International Journal of Engineering \& Technology, $7(2.31)(2018) 252-254$
International Journal of Engineering \& Technology
SPC
Website: www.sciencepubco.com/index.php/IJET
Research paper

\title{
Improved Qos in Connection-Oriented Approach for TCP/IP Suite
}

\author{
Sakshi Garg $^{*}$, Purushottam Sharma ${ }^{2}$ \\ ${ }^{1}$ Amity School of Engineering and Technology, Amity University Uttar Pradesh, India. \\ ${ }^{2}$ Amity School of Engineering and Technology, Amity University Uttar Pradesh, India. \\ E-mail:psharma5@amity.edu \\ *Corresponding author E-mail:sakshijyotigarg@gmail.com
}

\begin{abstract}
The TCP suite has various game plan lacks so far as Bandwidth utilize, Exchange Speed and blockage over system are concerned. Blockage is a problem which occurs on shared systems whenever several people battle for access to similar assets (transfer speed, bandwidth, and channels). The paper examines the parcel re-transmission issues identified with the association arranged system as to QoS parameters. Maybe, a model is proposed in the past paper which is approved in reference to QoS parameters and further the proposed demonstrate is differentiated to the current most pertinent strategies to conquer the deficiencies and decreasing clog in the system. The paper talks about the four most prime qualities i.e. reliability, delay, jitter and bandwidth and they are advocated based on the proposed model with the end goal that the recommended approach is giving an enhanced QoS. Moreover, the model is recommended gives $\mathrm{O}(\mathrm{n})$ as the time many-sided quality instead of the present systems which gives it as $\mathrm{O}(\mathrm{n} 2)$; given that the hypothetically computed multifaceted nature is the best and critical diminishment to the current ones.
\end{abstract}

Keywords: Connection-oriented mechanism, TCP/IP, packet re-transmission, Quality of Service (QoS), congestion, bandwidth, jitter, delay, reliability.

\section{Introduction}

TCP/IP frameworks, for instance, the Internet are especially frail to obstruct because of their principal affiliation less nature. There are no virtual circuits with guaranteed information transmission. Packs are implanted by any host at whatever point, and those packages are variable in gauge, which make expecting movement outlines and giving guaranteed benefit shocking. [2] Whereas connectionless systems have favorable circumstances, quality of service isn't one of them. The capacity of a system is to supply better help for good service over various systems administration innovations known as Quality of Service (QoS). The fundamental objectives of QoS are to assure the needs like controlled delay, submitted data transmission, jitter and reliability. [3][4]

Huge endeavors are being made by the Internet Engineering Task Force (IETF) to improve the service model of the Internet to help coordinated services for voice, video, and information transport. The adaptability, execution, and security of organized frameworks depend in huge part on the arrangement and administration of framework state, including both the portion level and applicationlevel state used to give a service. [6] Selective Repeat ARQ system is a technique to guarantee the solid conveyance of parce yet does not take dropped bundles into concern gave that the drop is because of whatever variables. [9] This paper along these lines gives the defense of the technique proposed in the past paper, as far as QoS parameters and furthermore discusses the future work on the real time information to deal with this glitch in the existing system.

The paper on a very basic level focuses on contemporary approach for the re-transmission of information to guarantee Quality of Service (QoS) without making clog on the system, ensuring highest bandwidth utilization, more reliability and robustness.
Additionally, Section II gives the Literature Study for the proposed model. In Section III, projected approach for the proposed model and details of the existing related techniques and the proposed work to be done is discussed. Section IV talks about the Qos parameters for the proposed methodology. Section V examines the outcomes and Section VI contains the Conclusions. Finally, Section VII demonstrates the Future Proceedings.

\section{Literature Study}

A.S Abdelrahman et al., gave a superior comprehension of the hidden layers of the system and check whether and where changes can be made. In execution arrange the Continuous Transport Convention (RTP) bundles for VoIP applications had been sent and contrasted and TCP/UDP parcels to acquire comes about which are chiefly identified with Quality of Service (QoS) factors. The achieved result endorsed that RTP consider to be smarter to lessen a bundle misfortune than UDP and furthermore affirmed that UDP/RTP are most solid since they had a little postponement and jitter conversely with TCP. [1]

S. Iren et al., discussed transport layer conventions given to endto-end correspondence between at least two hosts. This paper introduced an instructional exercise on transport layer ideas and phrasing, and an overview of transport layer services and conventions. The vehicle layer convention TCP is utilized as a source of perspective point, and looked into with nineteen different conventions outlined in the course of recent decades. [2] A. Sheih et al., depicted customary working structure interfaces and system tradition executions propel some structure state to be kept on the two sides of an association. This state settling the association with its endpoints, blocked straightforward failover, 
permitted refusal of-advantage ambushes, and compelled flexibility. His article showed a novel TCP-like transport tradition and another interface to supplant associations that together connect with all state to be kept toward one side point, permitting the other endpoint, dependably the server, to work with no peralliance state called Streams. The tradition is secure against changing and replay strikes, and the customer interface is in reverse staggering, requiring no developments to associations based customer applications. [5]

Wasan Ali Hussein et al., analyzed the execution of transport layer traditions for media application in the wired system. More conclusively, TCP and UDP Execution were surveyed then examined.

Execution evaluated by the QoS estimations like throughput, package transport extent, decency, and end-to-end delay. This QoS basis had been resolved for each one of two transport tradition. System test system (NS-2.35) was used to reenact and actualize both of TCP and UDP.[7]

S. Garg et al., examines the bundle transmission issues related to the association less and association arranged traditions in the Transport Layer.

Hence, an approach is proposed for the overcoming of the shortcomings and extending transmission restrict and diminishing clog in the system and wins with respect to giving the proposed arrangement multifaceted nature as $\mathrm{O}(\mathrm{n})$ which is perfect, that can be in every practical sense achieved.[9]

\section{Projected Approach}

The algorithm drafted in the paper [9] based on the changes to the TCP/IP Header which is tried to be justified theoretically through this paper.

\section{Work in Proposed Model}

The proposed model is discussed in addition to the paper [9]. It is projected to collect real time data samples from an authentic source like the TRAI application and thereafter results shall be computed for the approach.

It is expected from the proposed algorithm that the model will ensure timely delivery of the packet and also reliability of the data.

Likewise, the samples will be evaluated on the packet loss count keeping other factors constant. Every data sample will be analyzed for its best case and worst case to ensure the efficiency. Further, decision trees will be used to derive the results and due comparison will be made of the proposed model results to the existing model in terms of space complexity, time complexity, bandwidth utilization, reliability and other QoS parameters. The best can be explained with the future paper containing the samples and the results of them

Though the theoretical justification is made in the preceding paper regarding the complexity being reduced to $\mathrm{O}(\mathrm{n})$ from $\mathrm{o}\left(\mathrm{n}^{2}\right)$, even in the worst case.

\section{Available Techniques}

In case of the existing models there are several other techniques that work on sure delivery of the packet but do not concern about the dropped packets like the Automatic Repeat reQuest (ARQ) technique.

\section{Automatic Repeat Request}

It (ARQ), generally called Automatic Repeat Query, is a mix up control system for information transmission that usages affirmations (messages sent by the recipient exhibiting that it has successfully gotten an information packaging or convey) timeouts (demonstrated time allotments allowed to sneak past before an affirmation is gotten) to achieve strong information transmission over conniving administration .

ARQ conventions can be arranged into Selective Repeat ARQ / Selective Reject, Go-Back-N ARQ and Stop-and-wait ARQ [3][4].

\section{Stop and Wait ARQ Protocol}

It is like way recommended as turning bit custom, is a system in media exchanges to send information between two related contraptions. It ensures that information isn't lost due to dropped groups and that social occasions are gotten in the correct demand. It is the littlest troublesome changed repeat requests (ARQ) instrument. Sender sends one edge at any given minute; it is a striking occasion of the general sliding window tradition with transmit and get window sizes in respect to one and more evident than one self-sufficiently. In the wake of sending each packaging, the sender doesn't send any further edges until the point that it gets an assertion (ACK) signal. In the wake of getting a liberal edge, the gatherer sends an ACK. If the ACK does not accomplish the sender before a particular time, known as the timeout, the sender sends a basically indistinguishable packaging yet again. The timeout starting is reset after each bundling transmission. The above lead is a noteworthy example of Stop-and-Wait. Nevertheless, honest to goodness executions, they contrast to address certain issues of blueprint.

It is wasteful emerged from different ARQs, in light of the way that the time between groups, if the ACK and the data are gotten plausibly, is twofold the improvement time (expecting the turnaround time can be zero). The throughput on the channel is a little measure of what it could be. To deal with this issue, one can send more than one package at any given minute with a more important development number and use one ACK for a set.

\section{Go Back-N ARQ Protocol}

It is a particular event of the changed rehash demands (ARQ) convention, in which the sending system continues sending different lodgings appeared by a window evaluate even without tolerating an insistence (ACK) distribute the beneficiary. It is a wonderful event of the general sliding window convention with the transmit window size of $\mathrm{N}$ and get window size of 1 . It can transmit $\mathrm{N}$ lodgings to the sidekick before requiring an ACK.

It is a more skilled usage of a relationship than Stop-and-hold up ARQ, since not at all like sitting tight for a confirmation for each bundle; the association is so far being utilized as gatherings are being sent. In that point of confinement, amidst the time that would by some methods be spent postponing, more packages are being sent. Regardless, this technique additionally acknowledges sending graphs different conditions - if any edge was lost or hurt, or the ACK remembering them was lost or hurt, by then that edge and every last after edge in the window (regardless of whether they were gotten without goof) will be re-sent.

\section{Selective Repeat ARQ Protocol}

It is a particular occasion of the Automatic Repeat-Request (ARQ) tradition used to comprehend grouping number issue in interchanges.

It is a touch of the changed rehash demands (ARQ). With particular rehash, the sender sends different lodgings controlled by a window evaluate even without the need to sit tight for individual ACK from the gatherer as in Go-Back-N ARQ. The specialist may especially dismiss a lone edge, which might be retransmitted alone; these shows up diversely in connection to different sorts of ARQ, which must send each edge starting there yet again. The recipient perceives out-of-compose lodgings and sponsorships them. The sender independently retransmits follows that have formed out. 


\section{QoS Parameters}

The comparison between the proposed and the existing technique can be expressed in terms of time and space complexity where theoretically it has been justified that the proposed model is far more efficient in terms of complexity as it has been reduced from $\mathrm{O}\left(\mathrm{n}^{2}\right)$ to $\mathrm{O}(\mathrm{n})$ which is significantly huge reduction in terms of time complexity and that too, in the worst case.

Basically 4 types of characteristics are the most decisive ones: reliability, delay, jitter and bandwidth in QoS paradigms. [8]

\section{Reliability}

The proposed model ensures reliability as the objective of the proposed technique is to provide sure delivery of the dropped packets also such that the bandwidth can be utilized efficiently to its maximum.

\section{Delay}

Since, this approach is proposed to ensure highest bandwidth utilization, even in case of congestion in the network or the dropped packet scenario, the approach suggests holding back the troublesome transmission for a pseudo random time and making the bandwidth available for the next transmission making no delay to packet transmission therefore.

\section{Jitter}

When the packets are transmitted they are sent with the sequence number and their ordering and re-ordering is ensured at the source and destination end, so the proposed approach makes no interference to the existing ways of ensuring low jitter. Rather, when the packet will not discover delay in the transmission, the jitter is expected to be low and so the parameter of jitter in flow characteristics of QoS will be ensured.

\section{Bandwidth}

The aim of this approach is to ensure highest efficient bandwidth utilization such that there is sure delivery of the data packets in the network and the retransmitted packets are also sustained in the buffer and transmitted after a pseudo random time calculated according to the proposed algorithm in the earlier paper to provide maximum bandwidth for each transmission causing lesser congestion in the network and sure delivery.

\section{Results \& Discussions}

According to the proposed method the Time Complexity, is reduced to a great extent that is $\mathrm{O}(\mathrm{n})$ which is the best that can be practically achieved in the worst case as well. This technique can commit lesser congestion, higher efficiency, higher reliability, higher bandwidth utilization and robustness. Since there can be nnumber of such packets that has to be re-transmitted over the network the complexity theoretically come out to be $\mathrm{O}(\mathrm{n})$. This is the least Time Complexity one can achieve for this function for $n$ number of the packets to be retransmitted.

\section{Conclusion}

The present system considers selective packets for re-transmission but states no promise to reliable delivery of dropped packet in case of connection-oriented approach. The worst case scenario for the present system is $\mathrm{O}\left(\mathrm{n}^{2}\right)$ that is way too much to the theoretical technique proposed as it gives the worst case complexity also to be $\mathrm{O}(\mathrm{n})$.It is a significant reduction in the time as compared to currently available techniques. Thereafter, this can promise sure delivery of packets, very low jitter, higher bandwidth utilization, lesser delay and more reliability. It guarantees lesser congestion with a better time complexity ensuring improved Quality of Service.

\section{Future Proceedings}

The future aspects of the proposed work are to collect the real time data samples and justify the proposed work and validate the QoS parameters. Comparison with the existing techniques can be made to rationalize the proposed model and yield results in the future paper.

\section{References}

[1] Abdelrahman AS, Saeed RA \& Alsaqour RA, "QoS performance study of Real-Time Transport Protocol over VoIP", ARPN Journal of Engineering and Applied Sciences, Vol.11, No.9, (2016), pp.5608-5615.

[2] Iren S, Amer PD \& Conrad PT, "The transport layer: tutorial and survey", ACM Computing Surveys (CSUR), vol.31, no.4, (1999), pp.360-404.

[3] Leon-Garcia A \& Widjaja I, Communication networks. McGrawHill, Inc, (2003).

[4] Forouzan AB, Data communications \& networking (sie), Tata McGraw-Hill Education, (2006).

[5] Shieh A, Myers AC \& Sirer EG, "A stateless approach to connection-oriented protocols", ACM Transactions on Computer Systems (TOCS), vol.26, no.3, (2008).

[6] Wheeb AH, "Performance Comparison of Transport Layer Protocols", International Journal of Advanced Research in Computer Science and Software Engineering, vol.12, no.5, (2015), pp.121-125.

[7] Wasan AH, "Performance Comparison of Transport Layer Protocols for Multimedia Application in Wired Networks", IOSR Journal of Computer Engineering, vol.18, no. 6, (2016), pp.33-38.

[8] Congestion Control Mechanisms [online]. Available: http://www.linktionary.com/c/congestion.htm.

[9] Garg S, "A Novel Approach for Efficient Bandwidth Utilization in Transport Layer Protocols", International Journal of Research and Scientific Innovation (IJRSI), vol.3, no.4, (2018). 